\title{
Binuclear Cyclopentadienylcobalt Carbonyls: Comparison with Binuclear Iron Carbonyls
}

\author{
Hongyan Wang, ${ }^{\mathrm{a}, \mathrm{b}}$ Yaoming Xie, ${ }^{\mathrm{b}}$ R.Bruce King, ${ }^{\mathrm{b}}$ and Henry F. Schaefer III \\ Atomic and Molecular Physics Institute, Sichuan University \\ Sichuan 610065, P. R. China \\ and \\ Department of Chemistry and Center for Computational Chemistry \\ University of Georgia, Athens, Georgia 30602, USA
}

\section{Supporting Information}

Table S1. Harmonic vibrational frequencies (in $\mathrm{cm}^{-1}$ ) and infrared intensities (in parentheses, in $\mathrm{km} / \mathrm{mol}$ ) for the structure $\mathbf{I a}$ of $\mathrm{Cp}_{2} \mathrm{Co}_{2}(\mathrm{CO})_{3}$ with $\mathrm{C}_{2}$ symmetry

Table S2. Harmonic vibrational frequencies (in $\mathrm{cm}^{-1}$ ) and infrared intensities (in parentheses, in $\mathrm{km} / \mathrm{mol}$ ) for the structure $\mathbf{I b}$ of $\mathrm{Cp}_{2} \mathrm{Co}_{2}(\mathrm{CO})_{3}$ with $\mathrm{C}_{2}$ symmetry

Table S3. Harmonic vibrational frequencies (in $\mathrm{cm}^{-1}$ ) and infrared intensities (in parentheses, in $\mathrm{km} / \mathrm{mol}$ ) for the structure IIa of $\mathrm{Cp}_{2} \mathrm{Co}_{2}(\mathrm{CO})_{2}$ with $\mathrm{C}_{2 \mathrm{~h}}$ symmetry

Table S4. Harmonic vibrational frequencies (in $\mathrm{cm}^{-1}$ ) and infrared intensities (in parentheses, in $\mathrm{km} / \mathrm{mol}$ ) for the structure IIb of $\mathrm{Cp}_{2} \mathrm{Co}_{2}(\mathrm{CO})_{2}$ with $\mathrm{C}_{2 \mathrm{~h}}$ symmetry

Table S5. Harmonic vibrational frequencies (in $\mathrm{cm}^{-1}$ ) and infrared intensities (in parentheses, in $\mathrm{km} / \mathrm{mol}$ ) for the structure IIIa of $\mathrm{Cp}_{2} \mathrm{Co}_{2} \mathrm{CO}$ with $\mathrm{C}_{2 \mathrm{v}}$ symmetry

Table S6. Harmonic vibrational frequencies (in $\mathrm{cm}^{-1}$ ) and infrared intensities (in parentheses, in $\mathrm{km} / \mathrm{mol}$ ) for the structure IIIJ of $\mathrm{Cp}_{2} \mathrm{Co}_{2} \mathrm{CO}$ with $\mathrm{C}_{\mathrm{s}}$ symmetry

Figure S1. Structure of the $\mathrm{Cp}_{2} \mathrm{Co}_{2} \mathrm{CO}, \mathrm{Cp}_{2} \mathrm{Co}_{2}(\mathrm{CO})_{2}$, and $\mathrm{Cp}_{2} \mathrm{Co}_{2}(\mathrm{CO})_{3}$

Table S7. The Co-Co bond Distances(in $\AA$ ), total energies (E, in Hartree) and relative energies $(\Delta \mathrm{E}$, in $\mathrm{kcal} / \mathrm{mol})$. The number of imaginary vibrational vibrational frequencies (Nimg) for each structure is also listed.

Table S8. Harmonic vibrational frequencies (in $\mathrm{cm}^{-1}$ )and infrared intensities (in parentheses, in $\mathrm{km} / \mathrm{mol}$ ) for the structure IVa of $\mathrm{Fe}_{2}(\mathrm{CO})_{9}$ with $\mathrm{D}_{3 \mathrm{~h}}$ symmetry

Table S9. Harmonic vibrational frequencies (in $\mathrm{cm}^{-1}$ )and infrared intensities (in parentheses, in $\mathrm{km} / \mathrm{mol}$ ) for the structure $\mathbf{I V b}$ of $\mathrm{Fe}_{2}(\mathrm{CO})_{9}$ with $\mathrm{C}_{2 \mathrm{v}}$ symmetry

Table S10. Harmonic vibrational frequencies (in $\mathrm{cm}^{-1}$ )and infrared intensities (in parentheses, in $\mathrm{km} / \mathrm{mol}$ ) for the structure IVc of $\mathrm{Fe}_{2}(\mathrm{CO})_{9}$ with $\mathrm{C}_{2}$ symmetry

Figure S2. Structures computed for $\mathrm{Fe}_{2}(\mathrm{CO})_{9}$.

Complete Ref. 28 
Table S1. Harmonic vibrational frequencies (in $\mathrm{cm}^{-1}$ ) and infrared intensities (in parentheses, in $\mathrm{km} / \mathrm{mol}$ ) for the structure Ia of $\mathrm{Cp}_{2} \mathrm{Co}_{2}(\mathrm{CO})_{3}$ with $\mathrm{C}_{2}$ symmetry

\begin{tabular}{|c|c|c|c|c|c|c|c|c|c|}
\hline \multicolumn{3}{|c|}{ B3LYP } & \multicolumn{2}{|l|}{ BP86 } & \multicolumn{3}{|c|}{ B3LYP } & \multicolumn{2}{|l|}{ BP86 } \\
\hline $\mathrm{a}$ & 30 & (1) & 27 & (1) & $\mathrm{b}$ & 49 & (0) & 47 & $(0)$ \\
\hline & 43 & (0) & 40 & (0) & & 77 & (1) & 77 & (1) \\
\hline & 100 & (0) & 97 & (0) & & 109 & (0) & 109 & (0) \\
\hline & 104 & (0) & 102 & (0) & & 126 & (1) & 123 & (2) \\
\hline & 137 & (0) & 133 & (0) & & 127 & (1) & 129 & (0) \\
\hline & 189 & (0) & 190 & (0) & & 243 & (0) & 241 & (1) \\
\hline & 299 & (4) & 302 & (3) & & 305 & (6) & 306 & (6) \\
\hline & 332 & (3) & 333 & (3) & & 328 & (2) & 336 & (3) \\
\hline & 356 & (0) & 363 & (2) & & 351 & (7) & 354 & (1) \\
\hline & 442 & (0) & 447 & (0) & & 437 & (10) & 435 & (4) \\
\hline & 508 & (9) & 503 & (8) & & 499 & (4) & 498 & (12) \\
\hline & 533 & (11) & 532 & (11) & & 523 & (109) & 534 & (86) \\
\hline & 562 & (1) & 564 & $(0)$ & & 573 & (138) & 555 & (33) \\
\hline & 589 & (0) & 570 & (2) & & 579 & (14) & 561 & (10) \\
\hline & 595 & (1) & 574 & (1) & & 601 & (37) & 595 & (76) \\
\hline & 805 & (7) & 774 & (6) & & 614 & (133) & 615 & $(126)$ \\
\hline & 822 & (0) & 793 & (0) & & 802 & $(34)$ & 772 & (32) \\
\hline & 840 & (0) & 808 & (0) & & 822 & (127) & 794 & (106) \\
\hline & 842 & (0) & 812 & (0) & & 838 & (1) & 807 & (1) \\
\hline & 866 & (0) & 832 & (0) & & 844 & (6) & 813 & (1) \\
\hline & 912 & (0) & 872 & (0) & & 865 & (12) & 832 & (13) \\
\hline & 922 & (0) & 877 & (0) & & 912 & (1) & 873 & (0) \\
\hline & 1004 & (3) & 974 & (3) & & 922 & (10) & 877 & (13) \\
\hline & 1032 & (22) & 1000 & (21) & & 1004 & (10) & 974 & (9) \\
\hline & 1068 & $(0)$ & 1036 & $(0)$ & & 1032 & (4) & 1000 & (3) \\
\hline & 1072 & (1) & 1040 & (1) & & 1068 & (3) & 1037 & (3) \\
\hline & 1136 & $(0)$ & 1105 & $(0)$ & & 1071 & (4) & 1038 & (3) \\
\hline & 1273 & (0) & 1222 & (0) & & 1136 & (2) & 1105 & (4) \\
\hline & 1391 & (0) & 1349 & (0) & & 1273 & (0) & 1222 & (0) \\
\hline & 1397 & (0) & 1356 & (0) & & 1391 & (1) & 1348 & (1) \\
\hline & 1443 & (1) & 1389 & (1) & & 1396 & (18) & 1356 & (16) \\
\hline & 1476 & (2) & 1425 & (3) & & 1442 & (8) & 1389 & (9) \\
\hline & 1893 & $(583)$ & 1821 & $(477)$ & & 1476 & (2) & 1424 & (3) \\
\hline & 2052 & (2) & 1964 & (4) & & 2036 & (1415) & 1951 & (1090) \\
\hline & 3240 & (0) & 3165 & (0) & & 3240 & (1) & 3165 & (1) \\
\hline & 3245 & (0) & 3171 & (0) & & 3245 & (0) & 3171 & (0) \\
\hline & 3253 & (0) & 3178 & $(0)$ & & 3253 & (2) & 3178 & (2) \\
\hline & 3261 & (0) & 3187 & (0) & & 3261 & $(0)$ & 3187 & (0) \\
\hline & 3269 & (0) & 3194 & (0) & & 3269 & $(0)$ & 3194 & (1) \\
\hline
\end{tabular}


Table S2. Harmonic vibrational frequencies (in $\mathrm{cm}^{-1}$ )and infrared intensities (in parentheses, in $\mathrm{km} / \mathrm{mol}$ ) for the structure $\mathbf{I b}$ of $\mathrm{Cp}_{2} \mathrm{Co}_{2}(\mathrm{CO})_{3}$ with $\mathrm{C}_{2}$ symmetry

\begin{tabular}{|c|c|c|c|c|c|}
\hline \multicolumn{3}{|c|}{ BP86 } & \multicolumn{3}{|c|}{ BP86 } \\
\hline $\mathrm{a}$ & 24 & (0) & $\mathrm{b}$ & 20 & (0) \\
\hline & 61 & (2) & & 63 & (2) \\
\hline & 88 & (0) & & 88 & (0) \\
\hline & 115 & (0) & & 109 & (0) \\
\hline & 209 & (0) & & 212 & (0) \\
\hline & 215 & (0) & & 217 & (3) \\
\hline & 268 & (9) & & 267 & (1) \\
\hline & 303 & (1) & & 301 & (8) \\
\hline & 421 & (0) & & 334 & (20) \\
\hline & 437 & (0) & & 402 & (0) \\
\hline & 460 & (4) & & 431 & (0) \\
\hline & 484 & (0) & & 464 & (5) \\
\hline & 536 & (21) & & 543 & (21) \\
\hline & 563 & (0) & & 563 & (5) \\
\hline & 579 & (1) & & 580 & (4) \\
\hline & 783 & (0) & & 685 & (354) \\
\hline & 786 & (0) & & 784 & (40) \\
\hline & 804 & (7) & & 785 & (109) \\
\hline & 809 & (0) & & 803 & (2) \\
\hline & 819 & (0) & & 811 & (6) \\
\hline & 868 & (1) & & 818 & (4) \\
\hline & 874 & (1) & & 869 & (1) \\
\hline & 987 & (2) & & 874 & (3) \\
\hline & 993 & (16) & & 987 & (21) \\
\hline & 1038 & (0) & & 992 & (2) \\
\hline & 1042 & (1) & & 1038 & (0) \\
\hline & 1107 & (0) & & 1042 & (1) \\
\hline & 1224 & (0) & & 1107 & (3) \\
\hline & 1347 & (0) & & 1224 & (0) \\
\hline & 1354 & (0) & & 1347 & (0) \\
\hline & 1404 & (0) & & 1354 & (0) \\
\hline & 1410 & (5) & & 1404 & (2) \\
\hline & 1828 & (684) & & 1411 & (0) \\
\hline & 1883 & (0) & & 1829 & (701) \\
\hline & 3167 & (0) & & 3167 & (0) \\
\hline & 3168 & (0) & & 3168 & (0) \\
\hline & 3180 & (1) & & 3180 & (0) \\
\hline & 3182 & (0) & & 3182 & (0) \\
\hline & 3192 & $(0)$ & & 3192 & (2) \\
\hline
\end{tabular}


Table S3. Harmonic vibrational frequencies (in $\mathrm{cm}^{-1}$ ) and infrared intensities (in parentheses, in $\mathrm{km} / \mathrm{mol}$ ) for the structure IIa of $\mathrm{Cp}_{2} \mathrm{Co}_{2}(\mathrm{CO})_{2}$ with $\mathrm{C}_{2 \mathrm{~h}}$ symmetry

\begin{tabular}{|c|c|c|c|c|c|c|c|c|c|}
\hline & \multicolumn{2}{|c|}{ B3LYP } & \multicolumn{2}{|l|}{ BP86 } & & \multicolumn{2}{|c|}{ B3LYP } & \multicolumn{2}{|l|}{ BP86 } \\
\hline \multirow[t]{21}{*}{$a_{g}$} & 146 & $(0)$ & 147 & $(0)$ & $b_{g}$ & $11 \mathrm{i}$ & & $15 \mathrm{i}$ & \\
\hline & 191 & (0) & 195 & (0) & & 158 & $(0)$ & 153 & $(0)$ \\
\hline & 276 & (0) & 274 & (0) & & 371 & $(0)$ & 373 & $(0)$ \\
\hline & 379 & (0) & 374 & (0) & & 451 & (0) & 432 & $(0)$ \\
\hline & 424 & (0) & 422 & (0) & & 570 & (0) & 549 & $(0)$ \\
\hline & 493 & (0) & 491 & (0) & & 827 & (0) & 795 & (0) \\
\hline & 515 & (0) & 512 & (0) & & 844 & (0) & 808 & (0) \\
\hline & 593 & (0) & 566 & (0) & & 913 & (0) & 865 & $(0)$ \\
\hline & 828 & (0) & 796 & (0) & & 1019 & (0) & 987 & (0) \\
\hline & 839 & $(0)$ & 803 & $(0)$ & & 1069 & $(0)$ & 1037 & $(0)$ \\
\hline & 849 & (0) & 819 & (0) & & 1274 & (0) & 1223 & (0) \\
\hline & 919 & (0) & 873 & (0) & & 1387 & (0) & 1344 & $(0)$ \\
\hline & 1024 & (0) & 991 & $(0)$ & & 1452 & (0) & 1398 & $(0)$ \\
\hline & 1071 & (0) & 1040 & (0) & & 3242 & (0) & 3166 & (0) \\
\hline & 1138 & (0) & 1106 & (0) & & 3254 & (0) & 3177 & (0) \\
\hline & 1395 & (0) & 1356 & (0) & $\mathrm{b}_{\mathrm{u}}$ & 111 & (2) & 111 & (2) \\
\hline & 1459 & (0) & 1407 & $(0)$ & & 233 & (9) & 228 & (6) \\
\hline & 1900 & (0) & 1826 & (0) & & 322 & (23) & 322 & (20) \\
\hline & 3242 & $(0)$ & 3165 & $(0)$ & & 354 & (18) & 359 & (3) \\
\hline & 3259 & (0) & 3182 & (0) & & 531 & (10) & 527 & (15) \\
\hline & 3268 & (0) & 3191 & (0) & & 531 & (23) & 542 & (7) \\
\hline \multirow[t]{16}{*}{$\mathrm{a}_{\mathrm{u}}$} & 10 & (0) & $6 i$ & & & 633 & (40) & 641 & (80) \\
\hline & 37 & (2) & 35 & (2) & & 827 & (88) & 794 & (52) \\
\hline & 114 & (0) & 108 & (0) & & 839 & (17) & 804 & (21) \\
\hline & 346 & (8) & 349 & (8) & & 846 & (47) & 816 & (58) \\
\hline & 569 & (17) & 544 & (12) & & 917 & (5) & 871 & (9) \\
\hline & 592 & (1) & 564 & (0) & & 1024 & (18) & 991 & (18) \\
\hline & 842 & (1) & 805 & (0) & & 1069 & (8) & 1037 & (9) \\
\hline & 845 & (0) & 814 & (0) & & 1137 & (10) & 1105 & (12) \\
\hline & 913 & (1) & 865 & (1) & & 1390 & (15) & 1349 & (18) \\
\hline & 1019 & (26) & 987 & (25) & & 1459 & (4) & 1406 & (4) \\
\hline & 1073 & (0) & 1042 & (0) & & 1853 & (1189) & 1793 & (930) \\
\hline & 1274 & (0) & 1223 & (0) & & 3242 & (1) & 3165 & (1) \\
\hline & 1394 & (0) & 1355 & (0) & & 3259 & (0) & 3182 & (0) \\
\hline & 1452 & (6) & 1399 & (6) & & 3268 & (0) & 3191 & (1) \\
\hline & 3242 & (0) & 3166 & (1) & & & & & \\
\hline & 3254 & $(0)$ & 3177 & (1) & & & & & \\
\hline
\end{tabular}


Table S4. Harmonic vibrational frequencies (in $\mathrm{cm}^{-1}$ ) and infrared intensities (in parentheses, in $\mathrm{km} / \mathrm{mol}$ ) for the structure IIb of $\mathrm{Cp}_{2} \mathrm{Co}_{2}(\mathrm{CO})_{2}$ with $\mathrm{C}_{2 \mathrm{~h}}$ symmetry

\begin{tabular}{|c|c|c|c|c|c|c|c|c|c|}
\hline \multicolumn{3}{|c|}{ B3LYP } & \multicolumn{2}{|l|}{ BP86 } & \multicolumn{3}{|c|}{ B3LYP } & \multicolumn{2}{|l|}{ BP86 } \\
\hline$a_{g}$ & 95 & (0) & 92 & (0) & $b_{g}$ & 24 & (0) & 45 & (0) \\
\hline & 155 & (0) & 149 & (0) & & 109 & (0) & 113 & (0) \\
\hline & 228 & (0) & 217 & $(0)$ & & 249 & (0) & 272 & (0) \\
\hline & 288 & (0) & 294 & (0) & & 467 & (0) & 466 & (0) \\
\hline & 457 & (0) & 455 & (0) & & 601 & (0) & 587 & (0) \\
\hline & 488 & (0) & 515 & (0) & & 750 & (0) & 727 & (0) \\
\hline & 529 & (0) & 534 & (0) & & 816 & (0) & 782 & (0) \\
\hline & 637 & (0) & 623 & (0) & & 860 & (0) & 828 & (0) \\
\hline & 781 & (0) & 749 & (0) & & 988 & (0) & 956 & (0) \\
\hline & 811 & (0) & 772 & (0) & & 1053 & (0) & 1021 & (0) \\
\hline & 845 & (0) & 814 & (0) & & 1262 & (0) & 1210 & (0) \\
\hline & 927 & (0) & 886 & (0) & & 1356 & (0) & 1319 & (0) \\
\hline & 1012 & (0) & 983 & (0) & & 1412 & (0) & 1357 & (0) \\
\hline & 1067 & (0) & 1036 & (0) & & 3213 & (0) & 3138 & (0) \\
\hline & 1104 & (0) & 1071 & (0) & & 3231 & $(0)$ & 3155 & (0) \\
\hline & 1389 & (0) & 1348 & (0) & $\mathrm{b}_{\mathrm{u}}$ & 74 & (0) & 71 & (1) \\
\hline & 1441 & (0) & 1390 & (0) & & 188 & (26) & 185 & (25) \\
\hline & 2042 & (0) & 1951 & (0) & & 292 & (4) & 324 & (4) \\
\hline & 3219 & (0) & 3142 & (0) & & 381 & (13) & 383 & (5) \\
\hline & 3231 & (0) & 3153 & (0) & & 467 & (65) & 500 & (28) \\
\hline & 3245 & (0) & 3165 & (0) & & 502 & (24) & 510 & (21) \\
\hline$a_{u}$ & 45 & (2) & $5 i$ & & & 633 & (2) & 623 & (3) \\
\hline & 48 & (0) & 52 & (2) & & 760 & (53) & 734 & (49) \\
\hline & 109 & (1) & 100 & (1) & & 798 & (5) & 760 & (6) \\
\hline & 287 & (6) & 298 & (5) & & 837 & (31) & 809 & (32) \\
\hline & 451 & (5) & 452 & (3) & & 916 & (7) & 878 & (8) \\
\hline & 612 & (0) & 587 & (0) & & 1005 & (17) & 976 & (16) \\
\hline & 783 & (16) & 743 & (19) & & 1058 & (0) & 1029 & (0) \\
\hline & 816 & (1) & 784 & (1) & & 1102 & (5) & 1071 & (4) \\
\hline & 866 & (2) & 830 & (1) & & 1364 & (6) & 1329 & (6) \\
\hline & 991 & (28) & 960 & (28) & & 1441 & (1) & 1390 & (1) \\
\hline & 1060 & (0) & 1027 & $(0)$ & & 2025 & (2582) & 1939 & (1965) \\
\hline & 1262 & (0) & 1210 & (0) & & 3218 & (0) & 3141 & (1) \\
\hline & 1388 & (0) & 1341 & (0) & & 3231 & (0) & 3152 & (0) \\
\hline & 1414 & (6) & 1362 & (7) & & 3245 & (3) & 3165 & (5) \\
\hline & 3213 & (0) & 3138 & (1) & & & & & \\
\hline & 3231 & (4) & 3155 & (4) & & & & & \\
\hline
\end{tabular}


Table S5. Harmonic vibrational frequencies (in $\mathrm{cm}^{-1}$ ) and infrared intensities (in parentheses, in $\mathrm{km} / \mathrm{mol}$ ) for the structure IIIa of $\mathrm{Cp}_{2} \mathrm{Co}_{2} \mathrm{CO}$ with $\mathrm{C}_{2 \mathrm{v}}$ symmetry

\begin{tabular}{|c|c|c|c|c|c|c|c|c|c|}
\hline \multirow{2}{*}{$\mathrm{a}_{1}$} & \multicolumn{2}{|c|}{ B3LYP } & \multicolumn{2}{|l|}{ BP86 } & \multicolumn{3}{|c|}{ B3LYP } & \multicolumn{2}{|c|}{ BP86 } \\
\hline & 70 & (0) & 67 & (0) & $b_{1}$ & 24 & $(0)$ & 32 & (1) \\
\hline & 204 & (0) & 203 & $(0)$ & & 67 & $(0)$ & 65 & (1) \\
\hline & 339 & (6) & 345 & (7) & & 320 & (7) & 326 & (7) \\
\hline & 430 & (2) & 434 & (0) & & 455 & (4) & 432 & (1) \\
\hline & 521 & (13) & 524 & (10) & & 593 & (2) & 565 & (1) \\
\hline & 583 & (2) & 552 & (4) & & 817 & (1) & 777 & (0) \\
\hline & 805 & (5) & 773 & (1) & & 842 & (0) & 808 & (0) \\
\hline & 818 & (7) & 785 & (3) & & 889 & (2) & 842 & (1) \\
\hline & 843 & (0) & 812 & $(0)$ & & 1020 & (29) & 987 & (28) \\
\hline & 897 & (1) & 849 & (3) & & 1070 & (0) & 1040 & (0) \\
\hline & 1015 & (22) & 982 & (23) & & 1272 & $(0)$ & 1221 & (0) \\
\hline & 1068 & (1) & 1035 & (1) & & 1392 & (1) & 1356 & (0) \\
\hline & 1135 & (0) & 1102 & $(0)$ & & 1456 & (4) & 1404 & (4) \\
\hline & 1386 & (1) & 1344 & (1) & & 3238 & $(0)$ & 3160 & (0) \\
\hline & 1455 & (4) & 1401 & (4) & & 3251 & (2) & 3174 & (3) \\
\hline & 1971 & (786) & 1870 & $(581)$ & $\mathrm{b}_{2}$ & 79 & (25) & 126 & (4) \\
\hline & 3236 & (0) & 3161 & (0) & & 127 & $(0)$ & 176 & (5) \\
\hline & 3251 & (1) & 3173 & (2) & & 288 & $(0)$ & 299 & (1) \\
\hline & 3263 & (0) & 3186 & (0) & & 319 & (1) & 343 & (0) \\
\hline$a_{2}$ & 23 & (0) & 32 & (0) & & 418 & (11) & 432 & (4) \\
\hline & 148 & (0) & 148 & (0) & & 562 & (5) & 539 & (4) \\
\hline & 363 & (0) & 368 & (0) & & 802 & (111) & 771 & (88) \\
\hline & 576 & (0) & 554 & (0) & & 810 & (4) & 779 & (2) \\
\hline & 814 & (0) & 777 & (0) & & 831 & $(0)$ & 801 & (1) \\
\hline & 825 & (0) & 788 & (0) & & 898 & (1) & 849 & (3) \\
\hline & 885 & (0) & 838 & (0) & & 1015 & (1) & 981 & (0) \\
\hline & 1020 & (0) & 988 & (0) & & 1061 & (0) & 1027 & (0) \\
\hline & 1067 & (0) & 1036 & (0) & & 1134 & (18) & 1101 & (21) \\
\hline & 1272 & (0) & 1221 & (0) & & 1378 & (3) & 1332 & (2) \\
\hline & 1390 & (0) & 1351 & (0) & & 1453 & (1) & 1399 & (1) \\
\hline & 1455 & (0) & 1402 & (0) & & 3236 & (0) & 3161 & (0) \\
\hline & 3238 & (0) & 3159 & (0) & & 3251 & (0) & 3173 & (0) \\
\hline & 3251 & $(0)$ & 3174 & $(0)$ & & 3263 & (1) & 3185 & (1) \\
\hline
\end{tabular}


Table S6. Harmonic vibrational frequencies (in $\mathrm{cm}^{-1}$ ) and infrared intensities (in parentheses, in $\mathrm{km} / \mathrm{mol}$ ) for the structure IIIb of $\mathrm{Cp}_{2} \mathrm{Co}_{2} \mathrm{CO}$ with $\mathrm{C}_{\mathrm{s}}$ symmetry

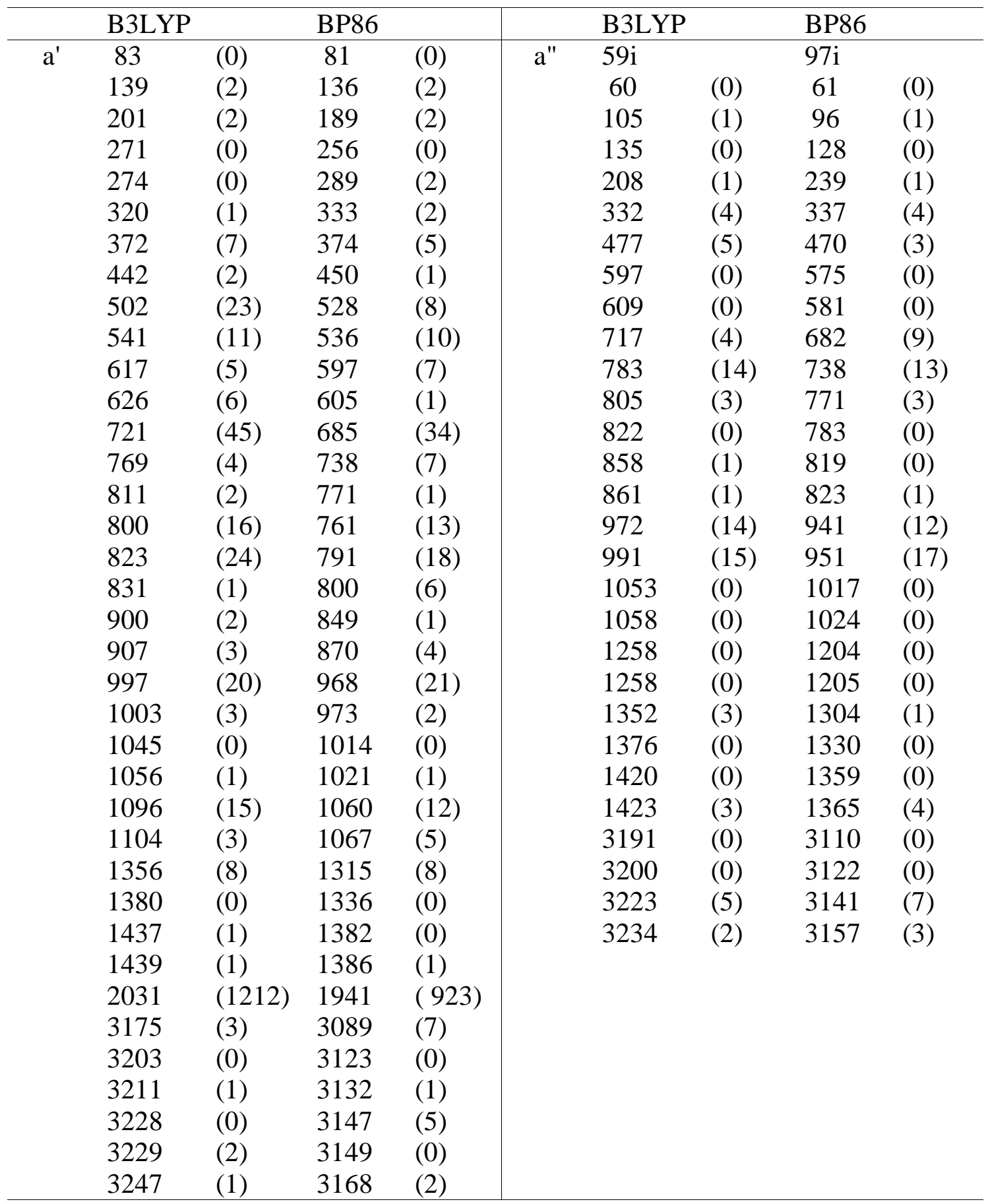



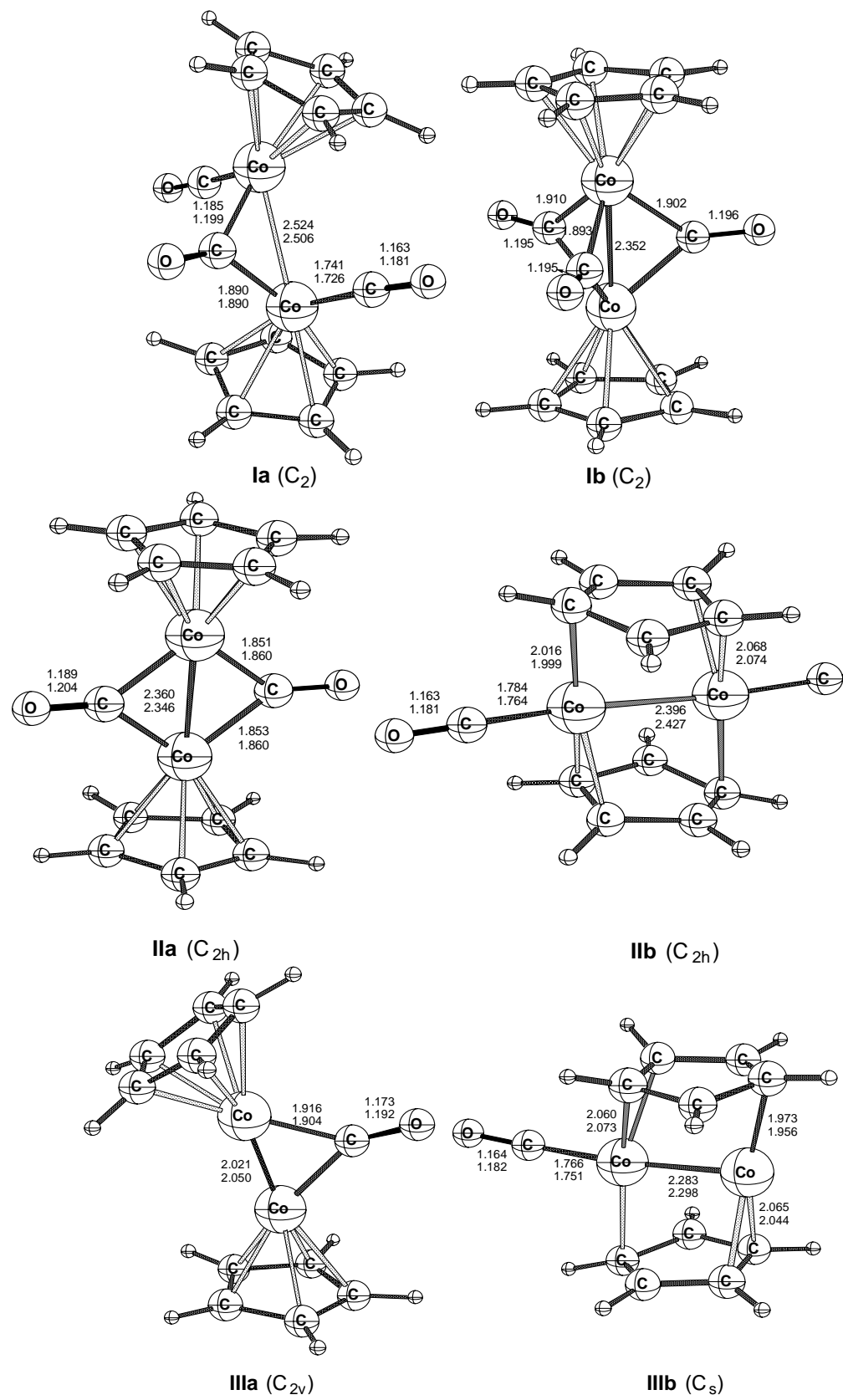

Figure S1. Structures computed for the $\mathrm{Cp}_{2} \mathrm{Co}_{2}(\mathrm{CO})_{n}(n=3,2,1)$ derivatives. 
Table S7. The Co-Co bond Distances(in $\AA$ ), total energies (E, in Hartree) and relative energies $(\Delta \mathrm{E}$, in $\mathrm{kcal} / \mathrm{mol})$. The number of imaginary vibrational vibrational frequencies (Nimg) for each structure is also listed.

\begin{tabular}{cccccc}
\hline & \multicolumn{2}{c}{$\mathrm{Cp}_{2} \mathrm{Co}_{2}(\mathrm{CO})_{3}$} & \multicolumn{2}{c}{$\mathrm{Cp}_{2} \mathrm{Co}_{2}(\mathrm{CO})_{2}$} \\
& & $\mathbf{I a}\left(\mathrm{C}_{2}\right)$ & $\mathbf{I b}\left(\mathrm{C}_{2}\right)$ & $\mathbf{I a}\left(\mathrm{C}_{2 \mathrm{~h}}\right)$ & $\mathbf{I I b}\left(\mathrm{C}_{2 \mathrm{~h}}\right.$ \\
\hline & $\mathrm{Co}-\mathrm{Co}$ & 2.524 & & 2.360 & 2.396 \\
\hline & $\mathrm{E}$ & -3492.86261 & & -3379.49682 & -3379.435 \\
\hline & $\Delta \mathrm{E}$ & 0 & & 0 & 38.2153 \\
\hline & $\mathrm{Nimg}$ & 0 & & $1(11 \mathrm{i})$ & 0 \\
\hline & $\mathrm{Co}-\mathrm{Co}$ & 2.506 & 2.352 & 2.346 & 2.427 \\
\hline & $\mathrm{E}$ & -3493.36137 & -3493.36424 & -3379.99660 & -3379.941 \\
& $\Delta \mathrm{E}$ & 1.80 & 0 & 0 & 34.86 \\
& $\mathrm{Nimg}$ & 0 & 0 & $1(15 \mathrm{i})$ & $1(5 \mathrm{i})$ \\
\hline
\end{tabular}


Table S8. Harmonic vibrational frequencies (in $\mathrm{cm}^{-1}$ )and infrared intensities (in parentheses, in $\mathrm{km} / \mathrm{mol}$ ) for the structure IVa of $\mathrm{Fe}_{2}(\mathrm{CO})_{9}$ with $\mathrm{D}_{3 \mathrm{~h}}$ symmetry

\begin{tabular}{|c|c|c|c|c|c|c|c|c|c|}
\hline \multirow[b]{2}{*}{$a_{1} T$} & \multicolumn{2}{|l|}{ B3LYP } & \multicolumn{2}{|l|}{ BP86 } & \multicolumn{3}{|c|}{ B3LYP } & \multicolumn{2}{|l|}{ BP86 } \\
\hline & 83 & (0) & 79 & (0) & $\mathrm{a}_{1} "$ & 54 & (0) & 51 & (0) \\
\hline & 259 & (0) & 255 & (0) & & 427 & $(0)$ & 417 & (0) \\
\hline & 397 & (0) & 399 & (0) & $a_{2} "$ & 98 & (0) & 93 & (0) \\
\hline & 466 & (0) & 478 & (0) & & 228 & (1) & 225 & (2) \\
\hline & 624 & (0) & 620 & (0) & & 434 & (52) & 451 & (18) \\
\hline & 957 & (0) & 1895 & (0) & & 583 & $(165)$ & 573 & (98) \\
\hline & 2170 & (0) & 2079 & (0) & & 697 & (763) & 675 & (798) \\
\hline$a_{2}^{\prime}$ & 82 & (0) & 77 & (0) & & 2120 & (2210) & 2038 & (1747) \\
\hline & 376 & (0) & 371 & (0) & $\mathrm{e}^{\prime \prime}$ & 55 & $(0)$ & 75 & $(0)$ \\
\hline & 525 & (0) & 508 & (0) & & 55 & (0) & 75 & (0) \\
\hline$e^{\prime}$ & 58 & (0) & 54 & $(0)$ & & 78 & (0) & 82 & (0) \\
\hline & 58 & (0) & 54 & (0) & & 78 & (0) & 82 & (0) \\
\hline & 88 & (0) & 85 & $(0)$ & & 159 & (0) & 159 & (0) \\
\hline & 88 & (0) & 85 & (0) & & 159 & (0) & 159 & (0) \\
\hline & 110 & (1) & 107 & (1) & & 313 & (0) & 314 & (0) \\
\hline & 110 & (1) & 107 & (1) & & 313 & (0) & 314 & (0) \\
\hline & 374 & (1) & 376 & (0) & & 443 & (0) & 452 & (0) \\
\hline & 374 & (1) & 376 & (0) & & 443 & (0) & 452 & (0) \\
\hline & 438 & (7) & 433 & (1) & & 491 & (0) & 486 & (0) \\
\hline & 438 & (7) & 433 & (1) & & 491 & (0) & 486 & (0) \\
\hline & 455 & (40) & 461 & $(25)$ & & 617 & (0) & 604 & (0) \\
\hline & 455 & (40) & 461 & (25) & & 617 & (0) & 604 & (0) \\
\hline & 545 & (10) & 532 & (4) & & 2096 & (0) & 2008 & (0) \\
\hline & 545 & (10) & 532 & (4) & & 2097 & (0) & 2008 & (0) \\
\hline & 545 & (10) & 532 & (4) & & & & & \\
\hline & 545 & (10) & 532 & (4) & & & & & \\
\hline & 631 & (120) & 624 & (134) & & & & & \\
\hline & 631 & (120) & 624 & (134) & & & & & \\
\hline & 1918 & (899) & 1870 & $(682)$ & & & & & \\
\hline & 1918 & (899) & 1870 & (682) & & & & & \\
\hline & 2101 & (1527) & 2013 & (1322) & & & & & \\
\hline & 2101 & (1528) & 2013 & $(1322)$ & & & & & \\
\hline
\end{tabular}


Table S9. Harmonic vibrational frequencies (in $\mathrm{cm}^{-1}$ )and infrared intensities (in parentheses, in $\mathrm{km} / \mathrm{mol}$ ) for the structure $\mathbf{I V b}$ of $\mathrm{Fe}_{2}(\mathrm{CO})_{9}$ with $\mathrm{C}_{2 \mathrm{v}}$ symmetry

\begin{tabular}{|c|c|c|c|c|c|c|c|c|c|}
\hline \multirow{12}{*}{$\mathrm{a}_{1}$} & B3LYP & & BP86 & & \multirow[b]{2}{*}{$\mathrm{b}_{1}$} & \multicolumn{2}{|c|}{ B3LYP } & \multicolumn{2}{|l|}{ BP86 } \\
\hline & 50 & (0) & 46 & $(0)$ & & 66 & $(0)$ & 64 & $(0)$ \\
\hline & 69 & (0) & 67 & $(0)$ & & 94 & $(0)$ & 89 & $(0)$ \\
\hline & 98 & (0) & 94 & (0) & & 104 & (1) & 101 & (1) \\
\hline & 119 & (0) & 113 & (0) & & 125 & (0) & 120 & (0) \\
\hline & 182 & (1) & 185 & (0) & & 359 & (0) & 360 & (0) \\
\hline & 366 & (0) & 370 & (0) & & 413 & (0) & 414 & (0) \\
\hline & 408 & (1) & 397 & (0) & & 469 & $(25)$ & 483 & (6) \\
\hline & 421 & (1) & 443 & (5) & & 559 & (6) & 543 & (5) \\
\hline & 441 & $(15)$ & 444 & (3) & & 571 & $(15)$ & 556 & (4) \\
\hline & 462 & $(0)$ & 463 & (2) & & 613 & (133) & 610 & $(144)$ \\
\hline & 486 & (5) & 498 & (0) & & 2101 & (2336 & 2011 & (1996 \\
\hline & & & & & & & ) & & ) \\
\hline & 645 & (132) & 642 & (49) & $b_{2}$ & 43 & (0) & 38 & (0) \\
\hline & 649 & $(24)$ & 647 & (99) & & 54 & (0) & 54 & (0) \\
\hline & 1909 & $(543)$ & 1850 & (459) & & 104 & (0) & 100 & (0) \\
\hline & 2078 & (1411 & 1999 & (1086 & & 108 & (1) & 106 & (0) \\
\hline & & ) & & ) & & & & & \\
\hline & 2096 & (329) & 2014 & $(362)$ & & 240 & (12) & 241 & (5) \\
\hline & 2171 & $(11)$ & 2086 & $(7)$ & & 387 & (105) & 388 & (14) \\
\hline$a_{2}$ & -18 & (0) & -21 & (0) & & 407 & $(56)$ & 433 & (126) \\
\hline & 81 & $(0)$ & 76 & $(0)$ & & 426 & (98) & 436 & $(46)$ \\
\hline & 94 & (0) & 89 & (0) & & 443 & (38) & 453 & (11) \\
\hline & 116 & (0) & 111 & (0) & & 476 & (1) & 481 & (19) \\
\hline & 375 & (0) & 378 & (0) & & 501 & (79) & 506 & (92) \\
\hline & 424 & (0) & 426 & (0) & & 638 & (322) & 633 & $(280)$ \\
\hline & 499 & (0) & 513 & (0) & & 664 & (86) & 664 & (126) \\
\hline & 557 & (0) & 543 & (0) & & 2060 & $(29)$ & 1983 & (39) \\
\hline & 599 & (0) & 591 & (0) & & 2080 & $(483)$ & 1998 & $(315)$ \\
\hline & 2070 & (0) & 1981 & (0) & & 2112 & (1466 & 2037 & (1259 \\
\hline
\end{tabular}


Table S10. Harmonic vibrational frequencies (in $\mathrm{cm}^{-1}$ )and infrared intensities (in parentheses, in $\mathrm{km} / \mathrm{mol}$ ) for the structure $\mathbf{I V c}$ of $\mathrm{Fe}_{2}(\mathrm{CO})_{9}$ with $\mathrm{C}_{2}$ symmetry

\begin{tabular}{|l|l|l|l|l|l|}
\hline & B3LYP & & & B3LYP & \\
\hline a & 22 & $(0)$ & b & 44 & $(1)$ \\
\hline & 45 & $(0)$ & & 56 & $(0)$ \\
\hline & 67 & $(0)$ & & 76 & $(0)$ \\
\hline & 84 & $(0)$ & & 94 & $(0)$ \\
\hline & 93 & $(0)$ & & 104 & $(1)$ \\
\hline & 99 & $(0)$ & 105 & $(1)$ \\
\hline & 108 & $(0)$ & & 109 & $(1)$ \\
\hline & 114 & $(0)$ & 09 & $(0)$ \\
\hline & 187 & $(0)$ & & 238 & $(13)$ \\
\hline & 367 & $(0)$ & 362 & $(6)$ \\
\hline & 376 & $(0)$ & 382 & $(110)$ \\
\hline & 391 & $(0)$ & & 400 & $(9)$ \\
\hline & 421 & $(0)$ & & 417 & $(31)$ \\
\hline & 423 & $(0)$ & 426 & $(59)$ \\
\hline & 441 & $(19)$ & 441 & $(2)$ \\
\hline & 463 & $(1)$ & & 473 & $(30)$ \\
\hline & 485 & $(8)$ & & 476 & $(2)$ \\
\hline & 499 & $(2)$ & 516 & $(32)$ \\
\hline & 561 & $(0)$ & & 560 & $(7)$ \\
\hline & 596 & $(8)$ & & 571 & $(37)$ \\
\hline & 642 & $(85)$ & & 611 & $(170)$ \\
\hline & 645 & $(53)$ & & 628 & $(406)$ \\
\hline & 1907 & $(545)$ & & 660 & $(93)$ \\
\hline & 2065 & $(4)$ & & 2064 & $(19)$ \\
\hline & 2080 & $(1603)$ & & 2082 & $(402)$ \\
\hline & 2093 & $(217)$ & & 2094 & $(2172)$ \\
\hline & 2170 & $(6)$ & & 2113 & $(1759)$ \\
\hline & & & & \\
\hline & & & & \\
\hline & & & & & \\
\hline
\end{tabular}




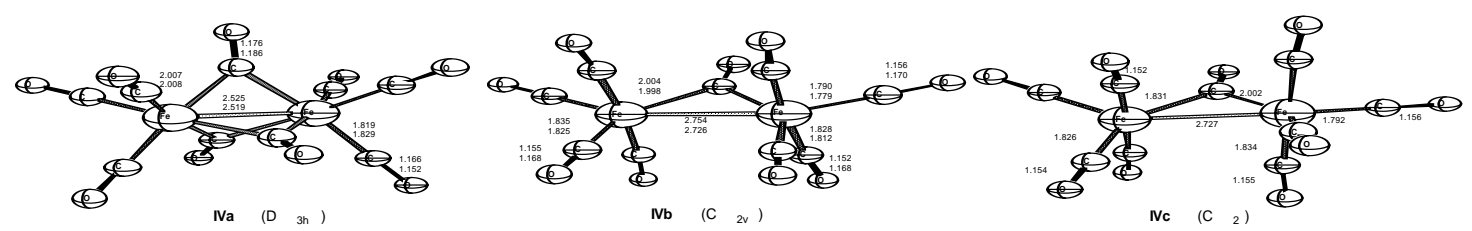

Figure S2. Structures computed for $\mathrm{Fe}_{2}(\mathrm{CO})_{9}$. 
Complete Ref. 28 : Frisch, M. J.; Trucks, G. W.; Schlegel, H. B.; Gill, P. M. W.; Johnson, B. G.; Robb, M. A.; Cheeseman, J. R.; Keith, T.; Petersson, G. A.;

Montgomery, J. A.; Raghavachari, K.; Al-Laham, M. A.; Zakrzewski, V. G.; Ortiz, J. V.;

Foresman, J. B.; Peng, C. Y.; Ayala, P. Y.; Chen, W.; Wong, M. W.; Andes, J. L.;

Replogle, E. S.; Gomperts, R.; Martin, R. L.; Fox, D. J.; Binkley, J. S.; Defrees, D. J.; Baker, J.; Stewart, J. J. P.; Head-Gordon, M.; Gonzalez, C.; Pople, J. A. Gaussian 94, Revision B.3; Gaussian Inc.: Pittsburgh, PA, 1995. 